in Study 2). Moreover, the present work also tested whether arousal could moderate the manifestation of positivity effect in Chinese older adults, given their preference for low-arousal positive emotions. Method: Participants were instructed to view 5 different types of stimulus (namely, neutral pictures, high arousal positive pictures, low arousal positive pictures, high arousal negative pictures, and low arousal negative pictures), and their recognition memories for these pictures were later tested in both experiments. Results: Significant age $x$ picture type interaction was found in both experiments, such that older Chinese adults underperformed their younger counterparts in recognizing high arousal positive pictures, as well as negative pictures regardless of arousal, but performed equally well (if not better) in recognizing low arousal positive pictures, which confirmed our original hypothesis. Conclusion: The present work highlights the importance of arousal in modulating emotional memory in Chinese older adults.

\section{REGIONAL DISPARITIES IN HEALTH AND HEALTH BEHAVIORS OF COSTA RICAN OLDER ADULTS}

E. Valdivieso ${ }^{1}, M$. Ivanisevic ${ }^{1}$, D. Johnson ${ }^{2}, 1$. University of Kansas, 2. UC Davis Alzheimer's Disease Center-East Bay

According to the WHO (2015), Costa Rica (CR) has the highest healthy life expectancy of the Central American region, being just second to Chile among all Latin American countries. However, important cultural and economic region disparities within the country exist (Human Development Index, 2017). Poverty, low education and other social determinants pose a risk factor for rural older adults' health. In this study, we compared rural (Guanacaste) and urban (San Jose) older adults on different health indicators: reported chronic illnesses, BMI, sleep quality (PSQI), and community participation. Participants were part of the Epidemiology and Development of Alzheimer's Disease (EDAD) study, which used a convenience sample of 311 cognitively healthy older adults (65 years and older). Results show that rural older adults had significantly more chronic conditions for $(\mathrm{M}=4.80, \mathrm{SD}=2.38)$ compared to urban dwellers $(\mathrm{M}=4.20$, $\mathrm{SD}=2.28), \mathrm{p}<.03$. Specifically, rural older adults had significantly higher rates of diabetes, heart disease, chronic back pain, kidney problems, musculoskeletal conditions, and sleep disturbances (for all, $\mathrm{p}<.05$ ). Urban older adults indicated higher frequencies of participation in community health activities compared to their rural counterparts $(\mathrm{p}=.02)$. No significant differences in BMI were found between the groups. These findings suggest that the study of healthy aging in Costa Ricans should be analyzed considering region as a social determinant of health. Public health policies in CR should aim at improving the social conditions associated to the poorer health status of rural older adults.

\section{SOCIAL CAPITAL AMONG BLACK SOUTH AFRICAN GRANDMOTHERS RAISING GRANDCHILDREN}

J. Muruthi ${ }^{1}$, M. Dolbin-MacNab ${ }^{2}$, S. Jarrott ${ }^{3}$, 1. Virgina Tech, 2. Virginia Polytechnic Institute and State University,

3. The Obio State University

As a result of the HIV/AIDS epidemic, unemployment and other sociocultural issues, grandmothers serve as primary caregivers for a significant number of Black South African children. Previous research has documented that Black South
African grandmothers experience significant physical, financial, emotional, and social adversity. However, less attention has been given to grandmothers' social support structures and the mechanisms by which these structures may help grandmothers cope with adversity. Guided by the social capital framework, the purpose of the present study was to explore the components of social capital (i.e., bridging, bonding, and linking) among 95 Black South African grandmothers raising grandchildren. Grandmothers participated in structured qualitative interviews during a weekly visit at a local luncheon (social) club. Results of a thematic analysis revealed that most grandmothers utilized all three types of social capital in order to promote individual and family well-being and survival. Bonding (e.g., close kinship ties) and bridging (e.g., network of neighbors and friends) capital were the most significant and common sources of immediate support. Specifically, family members provided emotional and instrumental support while neighbors and friends mainly provided instrumental support. Consistent with a social capital framework, some grandmothers described social ties as sources of stress that can affect health and function in the role. Grandmothers conceptualized linking capital (e.g., grants, organizational connections) as key resources that helped them access information, advice, and guidance. Participants were active agents in accessing and utilizing all three types of social capital. Implications of the findings for policy and practice will be addressed.

\section{SOCIAL SUPPORT AND SELF-RATED HEALTH AMONG OLDER ADULTS IN RURAL CHINA: DOES DISTINGUISHING PROVIDERS MATTER?}

\section{Y. Sun, M. Silverstein, Syracuse University}

There has been growing interest in the relationship between social networks and self-rated health in later life. However, less attention has been paid to the role of friends in this process. In particular, research in rural China rarely examines friends as sources of support, focusing more on family members who are critical for the well-being of older adults. This research uses data from the 2015 panel of the Longitudinal Study of Older Adults in Anhui Province to examine the association between social support and selfrated health, distinguishing support from family and friends. Further, psychological well-being is taken into account in as a mediator. Friends and family support networks were considered using measures from the Lubben Social Network Scale that indicate instrumental, affectual, and associational aspects of social networks. Regression models, with controls added for demographics, functional health, and diseases, reveal that social support from friends is positively associated with better self-rated health, and this effect is fully mediated by psychological well-being. No such relationship is found for support from relatives. That these findings were discovered in a region where family ties form the basis for much of social life in old age, highlights the importance of maintaining an active friendship network for improving self-perceptions of health.

\section{SOCIODEMOGRAPHIC AND HEALTH RELATED FACTORS ASSOCIATED WITH NUTRITIONAL STATUS IN THAI OLDER ADULTS}

A. Nawai ${ }^{1}$, S. Phongphangam ${ }^{2}$, S. Leveille ${ }^{3}, 1$. Boromarajonani College of Nursing, Chiang Mai, 2. University of Phayao, 3. University of Massachusetts Boston 